\title{
Swift J011511.0-725611: Discovery of a rare Be Star / White Dwarf binary system in the SMC
}

\author{
J. A. Kennea ${ }^{1 \star}$, M. J. Coe ${ }^{2}$, P. A. Evans ${ }^{3}$, L. J. Townsend ${ }^{4}$, Z. A. Campbell ${ }^{1}$, A. Udalski ${ }^{5}$ \\ ${ }^{1}$ Department of Astronomy and Astrophysics, The Pennsylvania State University, 525 Davey Lab, University Park, PA 16802, USA \\ ${ }^{2}$ Physics \& Astronomy, The University of Southampton, SO17 1BJ, UK \\ ${ }^{3}$ University of Leicester, X-ray and Observational Astronomy Research Group, School of Physics \& Astronomy, University Road, Leicester LE1 7RH, UK \\ ${ }^{4}$ South African Astronomical Observatory, P.O Box 9, Observatory, 7935, Cape Town, South Africa \\ ${ }^{5}$ Astronomical Observatory, University of Warsaw, Al. Ujazdowskie 4, 00-478 Warszawa, Poland
}

Accepted 2021 September 9. Received 2021 August 31; in original form 2021 July 29

\begin{abstract}
We report on the discovery of Swift J011511.0-725611, a rare Be X-ray binary system (BeXRB) with a White Dwarf (WD) compact object, in the Small Magellanic Cloud (SMC) by S-CUBED, a weekly X-ray/UV survey of the SMC by the Neil Gehrels Swift Observatory. Observations show an approximately 3 month outburst from Swift J011511.0-725611, the first detected by S-CUBED since it began in 2016 June. Swift J011511.0-725611 shows super-soft X-ray emission, indicative of a White Dwarf compact object, which is further strengthened by the presence of an $0.871 \mathrm{keV}$ edge, commonly attributed to O vIII K-edge in the WD atmosphere. Spectroscopy by SALT confirms the Be nature of the companion star, and long term light-curve by OGLE finds both the signature of a circumstellar disk in the system at outburst time, and the presence of a 17.4 day periodicity, likely the orbital period of the system. Swift J011511.0-725611 is suggested to be undergoing a Type-II outburst, similar to the previously reported SMC Be White Dwarf binary (BeWD), Swift J004427.3-734801. It is likely that the rarity of known BeWD is in part due to the difficulty in detecting such outbursts due to both their rarity, and their relative faintness compared to outbursts in Neutron Star BeXRBs.
\end{abstract}

Key words: stars: emission line, $\mathrm{Be}-\mathrm{X}$-rays: binaries

\section{INTRODUCTION}

Be/X-ray Binaries (BeXRBs) are high mass X-ray binaries (HMXB) consisting of typically a Neutron Star (NS) compact object and a main sequence star of B spectral type, showing strong emission line features in its optical spectrum, most commonly hydrogen Balmer lines, but also may show He and Fe emission lines (Hanuschik et al. 1996). Although NS are the most common compact object type in these objects, in some rare cases the compact object in the system has been reported to be a Black Hole (Munar-Adrover et al. 2014), or a White Dwarf (WD), most recently the WD containing BeXRB (hereafter BeWD) Swift J004427.3-734801 discovered in the SMC (Coe et al. 2020).

BeXRBs are often discovered through two modes of X-ray emission, typically referred to as Type-I and Type-II outbursts. Type-I outbursts $\left(L_{X} \approx 10^{36}-10^{37} \mathrm{erg} / \mathrm{s}\right)$, the most common, occur when the compact object interacts with the Be star. This accretion occurs when the compact object passes through a circumstellar disc (CSD) which forms around the Be star. The presence of the CSD is strongly inferred from the presence of excess infrared emission over that expected from the star, which brightens when the CSD grows Porter \& Rivinius (2003). Type-I X-ray outbursts are transient, and their onset are linked to the growth of the CSD (e.g. Kennea et al. 2020).

Type-II outbursts are typically at least an order of magnitude

^ E-mail: jak51@psu.edu (JAK) brighter than Type-I ( $\left.L_{\mathrm{X}} \gtrsim 10^{37} \mathrm{erg} / \mathrm{s}\right)$, sometimes reaching SuperEddington luminosities (e.g. in SMC X-3, Townsend et al. 2017), and are not obviously linked to the orbital period of the source, with outbursts that can span multiple orbital periods (Reig 2011). The extended length Type-II outbursts suggest the formation of an accretion disc around the compact object, but the conditions required to form this are unclear, with suggestion that warping of the CSD plays a role (e.g. Negueruela et al. 2001).

When the compact object is a NS, an X-ray pulsar is typically seen, along with an X-ray spectrum which in the soft X-ray band $(0.5-10 \mathrm{keV})$ is well described by a hard power-law spectrum with photon indices of $0-1$ typical. In the case of BeXRBs with WDs, the X-ray spectrum seen is more typical of a so-called Super Soft Source (SSS; e.g. Kahabka (2006)), in which the X-ray emission is thermal in nature and is predominantly emitting below $1 \mathrm{keV}$.

The irregular dwarf galaxy the Small Magellanic Cloud (SMC) lies at a distance of $62.1 \mathrm{kpc}$ (Graczyk et al. 2014), with a very low X-ray absorption in the line-of-sight of $N_{\mathrm{H}}=5.9 \times 10^{20} \mathrm{~cm}^{-2}$ (Dickey $\&$ Lockman 1990). Although many X-ray binaries are known in the SMC, all are HMXB, and predominantly they are BeXRBs, which has made it a well studied area of the sky to look for BeXRBs in the past (e.g. Haberl et al. 2000; Haberl \& Sturm 2016; Coe \& Kirk 2015).

Motivated by the study of BeXRBs, but with more of a focus on long term transient behaviour, the Swift SMC Survey (S-CUBED; Kennea et al. 2018) began in 2016. S-CUBED consists of a weekly 
survey of the optical extent of the SMC utilizing the Neil Gehrels Swift Observatory (Swift; Gehrels et al. 2004). The survey consists of 142 pointings with an exposure of 60 s each. Data are taken utilizing Swift's X-ray Telescope (XRT; Burrows et al. 2005) in Photon Counting (PC) mode and Ultra-violet/Optical Telescope (UVOT; Roming et al. 2005) utilizing the $u v w l$ filter. Due to the low background of XRT PC mode, 60s exposure allows for detection of an X-ray source in the SMC at a brightness of $1-2 \%$ Eddington Luminosity for a $1.4 \mathrm{M}_{\odot} \mathrm{NS}$ or brighter.

In this paper we report the discovery, by S-CUBED, of a likely new BeXRB containing a WD compact object, Swift J011511.0-725611. We present results of both S-CUBED and Swift target of opportunity (TOO) observations, as well as reporting on historical I-band lightcurve from the Optical Gravitation Lensing Experiment (OGLE; Udalski 2003; Udalski et al. 2015, and spectroscopic observations by the South African Large Telescope (SALT).

\section{OBSERVATIONS}

\subsection{Discovery of Swift J011511.0-725611}

Swift J011511.0-725611 was first discovered by the S-CUBED survey, and was reported by Kennea et al. (2021). The new source, internally designated SC1825 and subsequently named Swift J011511.0725611 using the standard Swift naming scheme, was first detected in S-CUBED data taken on 2020 December 29, with an initial brightness of $0.05_{-0.03}^{+0.07}$ count $\mathrm{s}^{-1}$. No previous detection of this source was found in any of the S-CUBED observations going back to when the survey started on 2016 June 8. Subsequent observations saw the brightness of the source rise to $0.15_{-0.03}^{+0.07}$ count s$^{-1}$ on 2021 January $5 \mathrm{~S}$-CUBED observation, and then $0.19_{-0.03}^{+0.08}$ count $\mathrm{s}^{-1}$ in the 2021 Jan 12 observation. At this point, a Swift Target of Opportunity (TOO) request was triggered to obtain more detailed observations.

The most accurate X-ray position for Swift J011511.0-725611, determined using the method described by Goad et al. (2007), was found to be R.A. (J2000) $=01^{h} 15^{m} 10.93^{s}$, Dec. $(\mathrm{J} 2000)=-72^{\circ} 56^{\prime} 10.4^{\prime \prime}$ with an estimated uncertainty of $2.0^{\prime \prime}$ radius (90\% confidence). In UVOT data we find a single bright s tar inside the X RT e rror circle. A catalog search of this source revealed a known star, 2MASS J01151085-7256102, with reported spectral types of O9IIIe (GoldenMarx et al. 2016) and O8-O9Ve (Lamb et al. 2013), suggesting that this source was a new BeXRB.

\subsection{Swift Observations}

The field containing Swift J011511.0-725611 has been regularly observed as part of the weekly S-CUBED survey (Kennea et al. 2018) starting on 2016 June 8th. As of 2021 July 27, it has been observed with S-CUBED on 199 occasions, with a median exposure time of 60 s per observation. Although S-CUBED observations are planned weekly, observation gaps occur frequently due to Swift visibility or scheduling constraints. In addition to the S-CUBED observations, detection of the outburst lead to TOO observations of Swift J011511.0-725611approximately every 1-2 days, starting on 2021 January 13 with the final exposure here taken on 2021 May 11. In addition, in 2021 July, late time observations totaling 5.08 ks exposure were taken post-outburst in order to determine a strong upper limit on quiescent emission from Swift J011511.0-725611. A detailed list of TOO observations is given in Table 1 and the measurements are shown in Fig. 1. For all XRT observations the data were collected in PC mode. UVOT data were collected in $u v w 1$ for S-CUBED data,

\begin{tabular}{|c|c|c|c|}
\hline ObsID & Begin (UTC) & End (UTC) & Exp. (s) \\
\hline 00013984001 & 2021-01-13 05:02:02 & 2021-01-13 08:30:54 & 4440 \\
\hline 00013984002 & 2021-01-14 20:47:02 & 2021-01-14 22:40:06 & 2000 \\
\hline 00013984003 & 2021-01-15 22:17:01 & 2021-01-16 01:41:26 & 2070 \\
\hline 00013984004 & 2021-01-16 19:04:02 & 2021-01-16 20:54:08 & 2095 \\
\hline 00013984005 & 2021-01-17 19:07:02 & 2021-01-17 22:26:46 & 1695 \\
\hline 00013984006 & 2021-01-27 02:40:02 & 2021-01-27 10:43:51 & 2150 \\
\hline 00013984010 & 2021-01-29 08:55:02 & 2021-01-30 12:19:00 & 2595 \\
\hline 00013984011 & 2021-01-31 11:43:02 & 2021-01-31 12:09:00 & 1355 \\
\hline 00013984012 & 2021-02-03 16:09:01 & 2021-02-03 16:39:01 & 1690 \\
\hline 00013984014 & 2021-02-05 03:20:02 & 2021-02-06 09:50:00 & 2030 \\
\hline 00013984015 & 2021-02-07 12:31:02 & 2021-02-07 12:58:57 & 1565 \\
\hline 00013984016 & 2021-02-10 21:41:01 & 2021-02-10 23:29:57 & 860 \\
\hline 00013984017 & 2021-02-11 04:03:02 & 2021-02-11 10:51:59 & 1920 \\
\hline 00013984019 & 2021-02-15 09:57:02 & 2021-02-15 10:27:01 & 1665 \\
\hline 00013984021 & 2021-02-18 00:00:02 & 2021-02-18 09:49:56 & 1290 \\
\hline 00013984022 & 2021-02-20 07:48:02 & 2021-02-20 09:50:57 & 1910 \\
\hline 00013984023 & 2021-02-22 09:11:01 & 2021-02-22 09:39:00 & 1575 \\
\hline 00013984024 & 2021-02-24 23:14:02 & 2021-02-24 23:44:01 & 1675 \\
\hline 00013984025 & 2021-02-26 08:43:02 & 2021-02-26 21:53:58 & 1730 \\
\hline 00013984026 & 2021-02-28 00:31:03 & 2021-02-28 19:57:01 & 1470 \\
\hline 00013984027 & 2021-03-02 08:17:02 & 2021-03-02 10:12:00 & 1895 \\
\hline 00013984028 & 2021-03-05 00:08:02 & 2021-03-05 05:01:58 & 1030 \\
\hline 00013984030 & 2021-03-26 01:32:14 & $2021-03-2608: 05: 57$ & 1380 \\
\hline 00013984031 & $2021-03-28$ 13:54:02 & 2021-03-28 14:22:58 & 1585 \\
\hline 00013984032 & 2021-03-30 18:22:02 & 2021-03-30 21:40:00 & 610 \\
\hline 00013984033 & 2021-03-31 05:44:02 & 2021-03-31 06:04:00 & 1135 \\
\hline 00013984034 & 2021-04-01 10:14:02 & 2021-04-01 10:38:58 & 1350 \\
\hline 00013984036 & 2021-04-05 16:02:20 & 2021-04-05 22:55:01 & 2045 \\
\hline 00013984037 & 2021-04-07 07:51:02 & 2021-04-07 22:15:00 & 730 \\
\hline 00013984038 & 2021-04-09 01:19:02 & 2021-04-09 01:48:56 & 1610 \\
\hline 00013984039 & 2021-04-11 20:20:27 & 2021-04-11 20:34:59 & 780 \\
\hline 00013984040 & 2021-04-13 02:47:02 & 2021-04-13 02:55:59 & 500 \\
\hline 00013984041 & 2021-04-15 18:16:02 & 2021-04-15 23:14:59 & 1860 \\
\hline 00013984042 & 2021-04-17 21:15:02 & 2021-04-17 23:03:56 & 2005 \\
\hline 00013984043 & 2021-04-21 00:00:02 & 2021-04-21 01:51:00 & 1961 \\
\hline 00013984044 & 2021-04-23 01:19:02 & 2021-04-23 22:19:01 & 2050 \\
\hline 00013984045 & 2021-04-25 13:42:02 & $2021-04-25$ 20:06:57 & 1515 \\
\hline 00013984046 & 2021-04-29 02:18:42 & 2021-04-29 04:03:00 & 860 \\
\hline 00013984047 & 2021-05-11 00:05:02 & 2021-05-11 09:49:57 & 1225 \\
\hline 00013984048 & 2021-07-07 00:08:02 & 2021-07-07 17:49:58 & 2100 \\
\hline 00013984049 & 2021-07-08 19:07:02 & 2021-07-08 23:59:59 & 390 \\
\hline 00013984050 & 2021-07-09 04:42:02 & 2021-07-10 20:30:01 & 1775 \\
\hline 00013984051 & 2021-07-13 21:54:02 & 2021-07-13 22:08:59 & 815 \\
\hline
\end{tabular}

Table 1. List of target of opportunity observations of Swift J011511.0725611 by Swift.

and in the all three UV filters for TOO observations. In addition some of the TOO observations also collected data in $u, b$ and $v$ filters. Although the TOO observations aimed for twice daily cadence, due to scheduling and spacecraft constraints, there are gaps in the coverage, especially during periods of time when the source was near the Swift orbit pole, when observations are not possible for up to 10 days.

We have analysed XRT and UVOT data from both Swift SCUBED observations and TOO observations, in order to produce long term light-curves and pre-outburst upper limits. Observations analysis was performed utilizing the standard Swift HEAsoft v6.28 tools, including xrtpipeline for XRT processing and extraction of spectra, and uvotmaghist for UVOT photometry. Extraction of XRT light-curves, include correction of detector issues such as hot pixels, hot columns and pile-up, was performed using the Python 


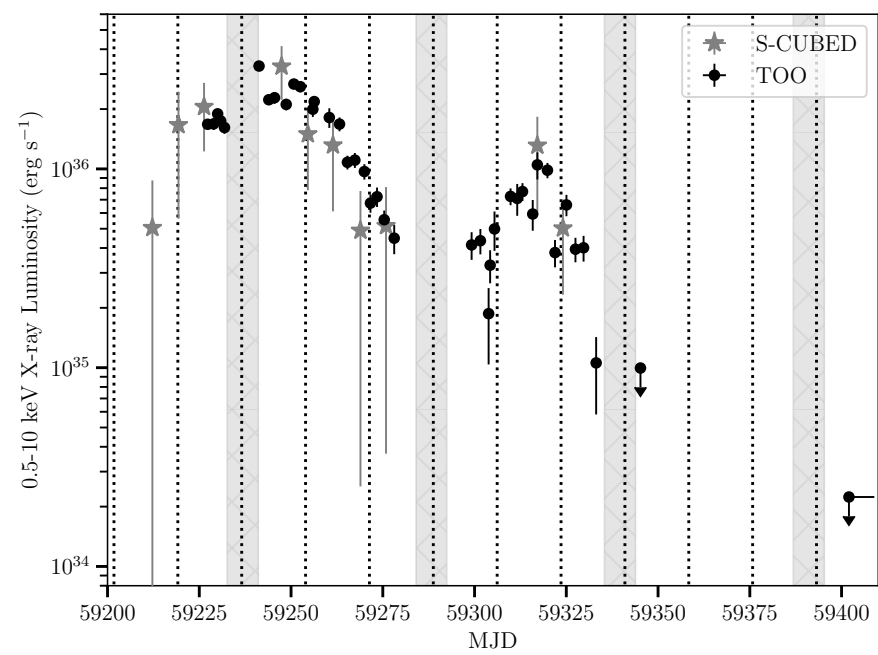

Figure 1. Combined S-CUBED and TOO X-ray light-curve of Swift J011511.0-725611, showing the outburst period. Grey regions show periods of time when the source was not observable by Swift due to observing constraints. Dotted lines show the optical period found from OGLE data, using Eqn. 1.

swifttools module ${ }^{1}$, which implements the methods presented by Evans et al. (2009). Spectral fitting was performed utilizing XSpec (Arnaud 1996).

The outburst light-curve is shown in Fig. 1, showing a double peaked outburst, with the observed brightness peaking on 2021 January 27 (MJD 59231.8), at a luminosity of $3.3 \pm 0.2 \times 10^{36} \mathrm{erg} \mathrm{s}^{-1}$ ( $0.5-10 \mathrm{keV})$, before fading, followed by a second period of outburst that peaked at a $1.0 \pm 0.2 \times 10^{36} \mathrm{erg} \mathrm{s}^{-1}(0.5-10 \mathrm{keV})$ around 2021 April 13 (MJD 59317.1). From initial detection by S-CUBED to the final detection in a TOO observation taken on 2021 April 29 (MJD 59333.1), the outburst is lasted approximately 120 days. Note that all luminosity measurements assume a standard SMC distance of $62.1 \mathrm{kpc}$ (Graczyk et al. 2014).

Following these two outburst peaks, the source faded to nondetection, and TOO observations ceased. S-CUBED monitoring of the source continued, but no detection of Swift J011511.0-725611 was made after the final upper TOO detection limit was measured on 2021 April 29. Combined late time observations taken in 2021 July find a $3 \sigma$ upper limit of $<2 \times 10^{34} \mathrm{erg} \mathrm{s}^{-1}(0.5-10 \mathrm{keV})$, showing that the quiescent $\mathrm{X}$-ray emission was at least 2 orders of magnitude fainter than the peak brightness. We note that Swift J011511.0725611 does not appear in any X-ray catalogue prior to discovery, despite being in fields observed by both Chandra and XMM-Newton in the past.

In order to obtain a high quality X-ray spectrum, we combined all the Swift XRT TOO data. The resultant spectrum is very soft, with the majority of X-ray emission below $2 \mathrm{keV}$ (see Fig. 2). This is in stark contrast to typical BeXRB in which the X-ray emission is typically a hard power-law with photon index $\simeq 1$ (Haberl \& Pietsch 2004). The very soft spectrum suggests that, analogous to the previously reported Swift J004427.3-734801, Swift J011511.0-725611 is a BeXRB with a WD compact object.

The X-ray spectrum is best fit by an absorbed blackbody model (Xspec bbodyrad), with mean $N_{\mathrm{H}}=0.194_{-0.024}^{+0.026} \times 10^{21} \mathrm{~cm}^{-2}$ (using

\footnotetext{
${ }^{1}$ https://pypi.org/project/swifttools/
}
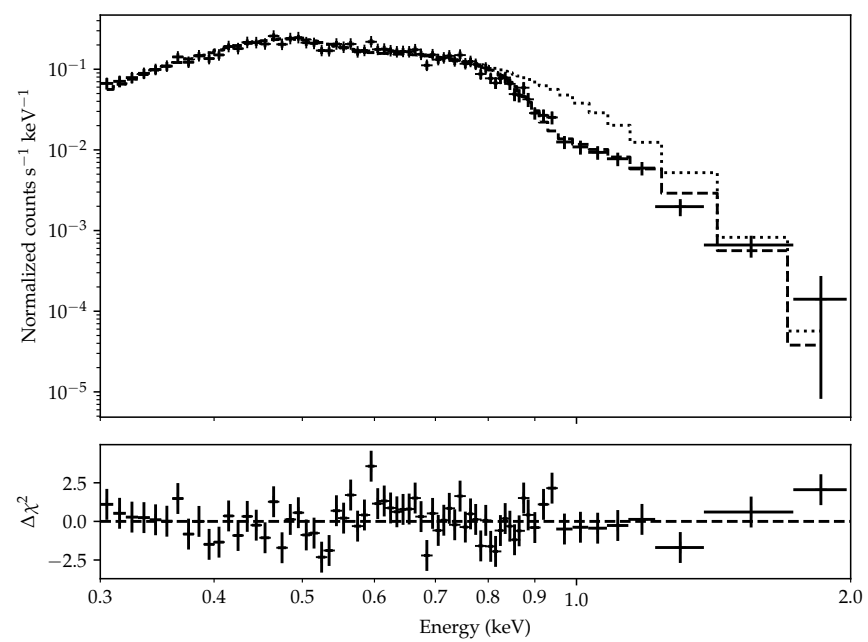

Figure 2. Combined X-ray spectrum of Swift J011511.0-725611 fit with an absorbed blackbody model, with an addition of an $0.871 \mathrm{keV} O$ viII absorption edge. The dashed line shows the fitted model without the edge component.

the Xspec TBabs absorption model with abundance set to wilm, e.g. Wilms et al. 2000). This absorption is higher than the expected level of $5.9 \times 10^{20} \mathrm{~cm}^{-2}$ (Dickey \& Lockman 1990), however we note that Swift J004427.3-734801 also showed enhanced absorption over the expected line-of-sight absorption (Coe et al. 2020), which may suggest localized absorption is a common feature in BeWD binaries. Mukai (2017) notes that excess absorption over line-of-sight is a common feature in accreting WD binaries.

The fit is significantly improved (reduced $\chi^{2}=0.95$ versus 2.14 ) by the inclusion of an absorption edge (using the Xspec edge model) with a fitted edge energy $E_{\text {Edge }}=0.864 \pm 0.011 \mathrm{keV}$, and an edge depth $\tau_{\text {Edge }}=1.84 \pm 0.32$. This is likely the signature of the $\mathrm{O}$ vIII $0.871 \mathrm{keV}$ absorption edge, a common feature of WD atmospheres frequently seen in SSS (e.g. Shimura 2000).

The fitted average black body temperature is $k T_{\mathrm{bb}}=96.7 \pm 4.2 \mathrm{eV}$. Assuming spherical accretion on the WD, Mukai (2017) shows that for a WD with mass $1.2 \mathrm{M}_{\odot}$, the maximum shock temperature would be $92 \mathrm{eV}$, consistent with the measured blackbody temperature in Swift J011511.0-725611. Therefore the X-ray emission here is consistent with an accreting WD scenario, with the mass of the WD of $\sim 1.2 \mathrm{M}_{\odot}$.

In order to make an estimate of the maximum radius of the emitting region, we fit the spectrum of Swift J011511.0-725611 at peak luminosity, with the same model as above, with $N_{\mathrm{H}}, E_{\text {Edge }}, \tau_{\text {Edge }}$, and $k T_{\mathrm{bb}}$ all fixed at the above average values, with only normalization free. The normalization is equal to $R_{\mathrm{bb}}^{2} / D_{10}^{2}$ where $R_{\mathrm{bb}}$ is the emission radius in kilometers, and $D_{10}$ is the distance to the source in units of $10 \mathrm{kpc}$, allowing emission radius to be calculated if the distance is known. From this fit we find a blackbody emission radius of $R_{\mathrm{bb}}=1642 \pm 83 \mathrm{~km}$, assuming the standard SMC distance of $62.1 \mathrm{kpc}$. This value is order-of-magnitude consistent with the estimated $\sim 4000 \mathrm{~km}$ radius of a $1.2 \mathrm{M}_{\odot}$ WD (e.g. Pringle \& Webbink 1975), consistent with the hypothesis that Swift J011511.0-725611 is a BeWD in the SMC, rather than a foreground object.

Analysis of the UVOT light-curve derived from both S-CUBED data and the TOO observations do not show any significant brightness changes in the optical/UV counterpart. Fig. 3 shows the S-CUBED Xray and UVOT $u v w 1$ light-curve for the entire period that S-CUBED 

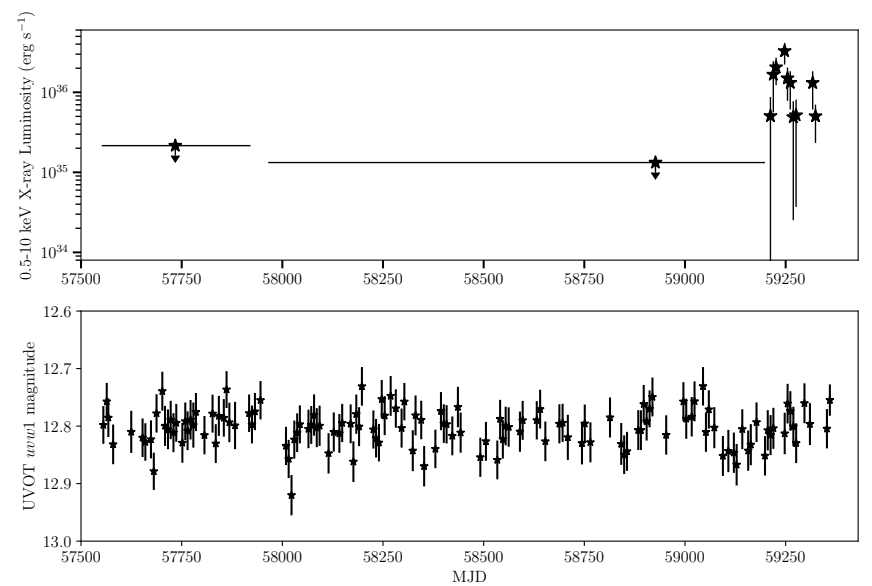

Figure 3. S-CUBED light-curve of Swift J011511.0-725611 showing all XRT and UVOT data. Upper limits on combined S-CUBED data taken before outburst are shown at a level of $\simeq 1-2 \times 10^{35} \mathrm{erg} \mathrm{s}^{-1}(0.5-10 \mathrm{keV})$. UVOT $u v w 1$ light-curve from S-CUBED is statistically consistent with no variability in the UV emission from Swift J011511.0-725611.

monitoring the source, starting in 2016 June. Significantly, the outburst of Swift J011511.0-725611 was not preceded or accompanied by any UV brightening. Fitting a constant level model to the UVOT $u v w 1$ light-curve reveals that it is consistent with no statistically significant $\mathrm{v}$ ariability, $\mathrm{w}$ ith $\mathrm{r}$ educed $\chi^{2}=0.9$. T herefore there is no detectable UV signature of this outburst.

\subsection{OGLE optical photometry}

The OGLE project (Udalski et al. 2015) undertakes to provide long term I-band photometry with an average cadence of 1-3 days. The star 2MASS J01151085-7256102 was observed continuously for nearly 2 decades in the I-band until COVID-19 restrictions prevented any further observations from March 2020. It is identified in the OGLE catalogue as:

O-III: smc115.1.38 I-band

O-IV: smc732.06.6370 I-band

OIII: smc115.1.v.17 V-band

OIV: smc732.06.v.7898 V-band

The I band data are shown in their entirety in Fig. 4. The overall behaviour revealed is consistent with that expected for a Be star in the SMC, showing large scale fluctuations on timescales of years. Also shown in this figure are the (V-I) colour changes observed by OGLE during this period. The source has clearly been much redder in colour since its return to its brightest state. This is discussed further in the Section 3 below.

Data from the most stable section of the OGLE observations, namely the 8 years after MJD 56500, were selected for a LombScargle timing analysis (Lomb 1976; Scargle 1982). The period range $2-100 \mathrm{~d}$ was investigated and one strong peak emerged at the period of $17.402 \mathrm{~d}$. The power spectrum for the range $10-25 \mathrm{~d}$ is shown in Fig. 5. The side peaks are the result of the true period beating with the annual sampling.

This subset of OGLE data was then folded at the period of 17.402 $\mathrm{d}$ and the resulting profile is shown in Fig. 6. Though there is some scatter in the individual cycles (as is shown in the lower panel of
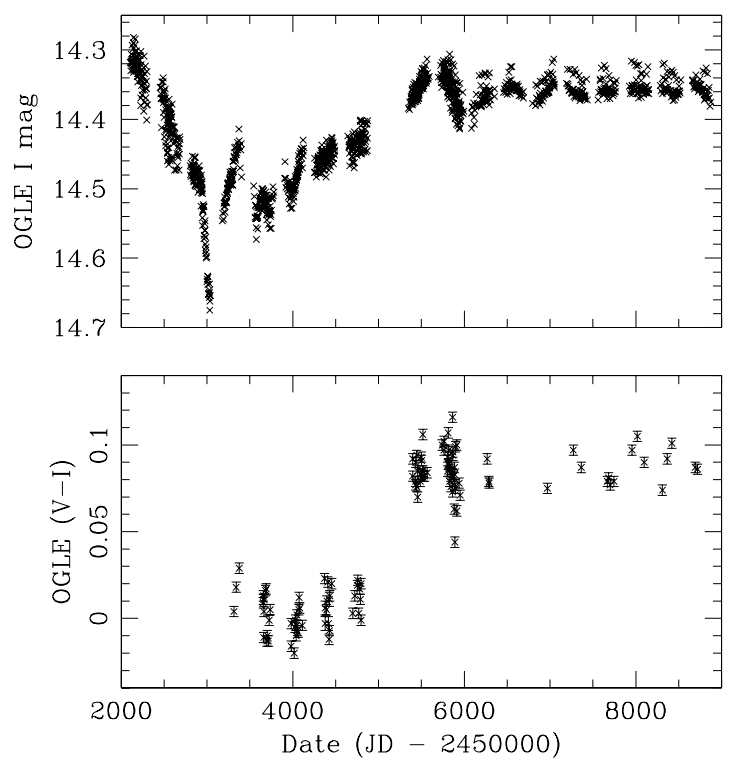

Figure 4. Top panel : OGLE III and IV observations of the optical counterpart to Swift J011511.0-725611. Lower panel OGLE (V-I) colour versus time.

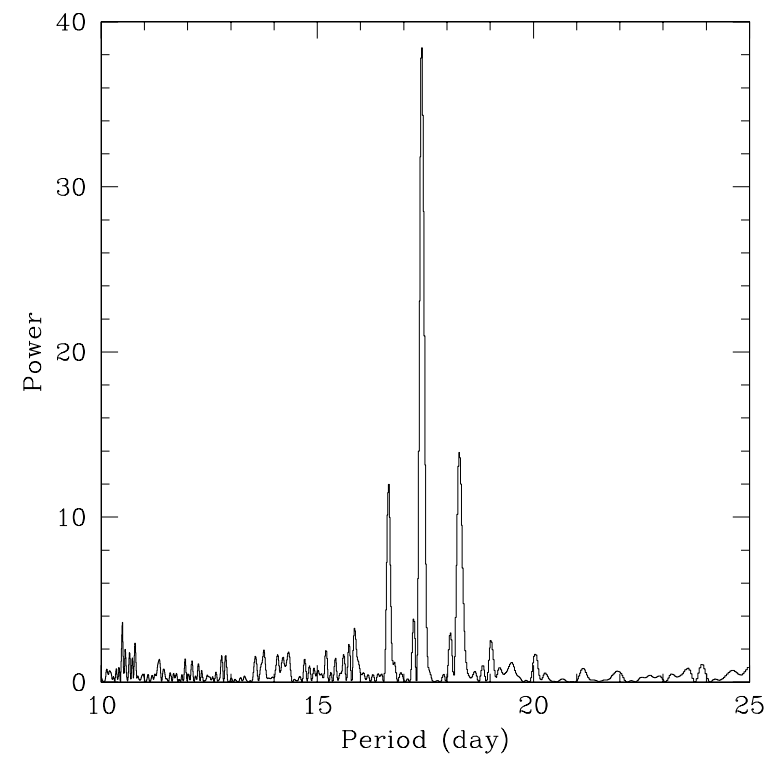

Figure 5. Lomb-Scargle power spectrum of OGLE data of Swift J011511.0725611. The main peak is at 17.402 days.

that figure) there clearly exists a dominant peak in the I-band light covering the phase range $\sim 0.3$ of a cycle, i.e. $\sim 5$ days.

The ephemeris for the time of the peak of the optical outbursts, $T_{\text {opt }}$, is:

$T_{\text {opt }}=56103.7+N(17.402) \mathrm{MJD}$

\subsection{SALT observations}

2MASS J01151085-7256102 was observed with the SALT on 2021 June 19 (JD 2459384.7) using the Robert Stobie Spectrograph (Burgh 

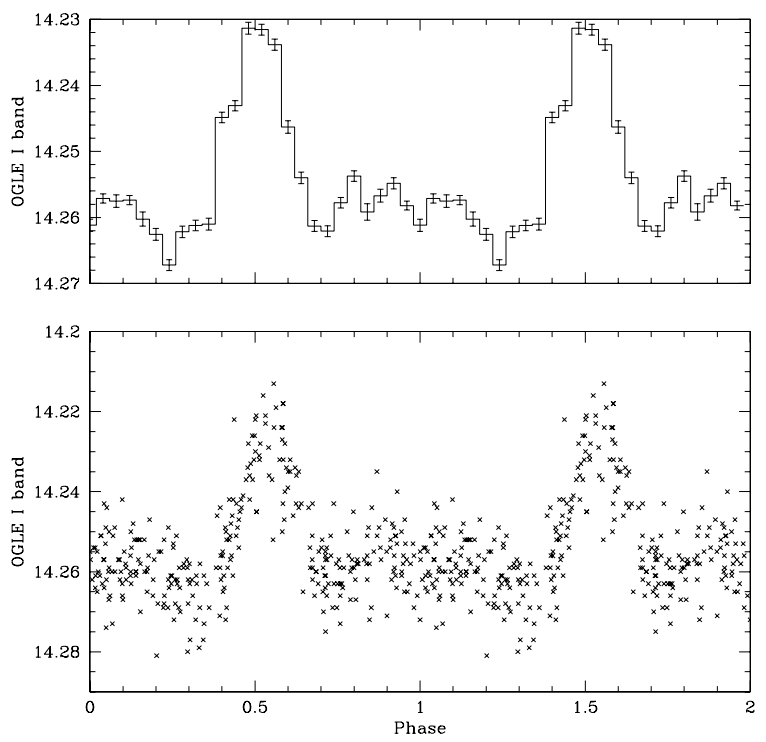

Figure 6. OGLE I-band data folded at the period of 17.402 days. Upper panel shows the averaged values for each phase bin. Lower panel shows the scatter in the measurements.
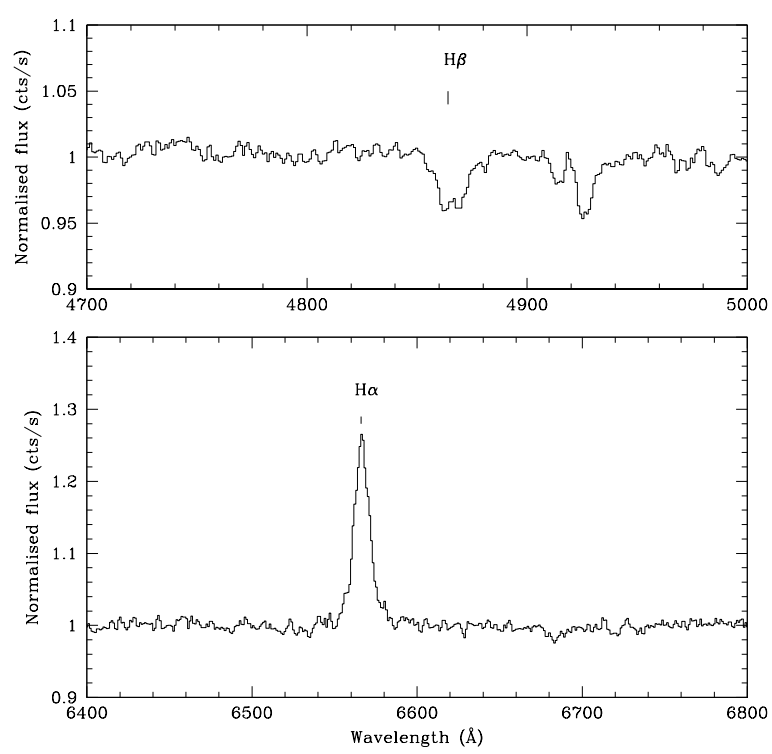

Figure 7. SALT spectra of 2MASS J01151085-7256102. The upper panel shows the $\mathrm{H} \beta$ profile, and the lower panel the $\mathrm{H} \alpha$ profile. In both cases the predicted position of the line is indicated, adjusted for the redshift to the SMC.

et al. 2003). The exposure time was $300 \mathrm{~s}$ and the grating used was PG0900. The PG0900 grating produces a spectrum over the range 4000-7000 $\AA$, at a resolution of approximately 1700 at $\mathrm{H}_{\alpha}$ and 1100 at $\mathrm{H}_{\gamma}$. The SALT observations of the two main hydrogen Balmer lines are shown in Fig. 7. $\mathrm{H} \alpha$ is clearly shown in emission, consistent with a single peak, and with an Equivalent Width of $-3.2 \pm 0.1 \AA$. The $\mathrm{H} \beta$ line is predominantly in absorption but with definite evidence of partial infilling. Such Balmer line emission is characteristic of the presence of a circumstellar disk around the B-type star.

The blue end of the spectral range is shown in Fig. 8. The spectrum shows prominent HeII lines at 4200, 4541, 4686 and $5412 \AA$, as well as the ionized $\mathrm{C}+\mathrm{O}$ blend at $4650 \AA$. These lines show that the star is a late-type O star. As outlined in Walborn \& Fitzpatrick (1990) and discussed in Golden-Marx et al. (2016), the ratio of HeII 4541 / HeI 4471 is $<1$, indicating a spectral type later than O7. The ratios HeII 4200 / HeI 4143 and HeII 4541 / HeI 4388 are both approximately 1 , signifying a spectral type of O9. This is supported by the clear identification of CIII 4650 (blended with the weaker OII line) and possible detection of SiIV 4089 (though we note it is very difficult to identify this line for certain above the noise of the continuum); both indicators of late-type $\mathrm{O}$ stars. It is difficult to distinguish between luminosity classes III-V due to the absence of strong metal lines in the SMC. However, our spectrum matches very well with the one presented in Golden-Marx et al. (2016) (see the bottom spectrum of their Fig. 8). Those authors conclude an O9 IIIe classification based partly on the V-magnitude expected at the distance of the SMC. Based on our spectrum, we fully agree with their classification.

\section{DISCUSSION}

Swift J011511.0-725611 like many other BeXRB systems was discovered through X-ray outburst, in this case the outburst itself was prolonged, much longer than the measured 17.4 day orbital period, suggesting a Type-II outburst. The soft thermal spectrum and presence of an $\mathrm{O}$ VIII absorption edge, commonly seen in WD sources such as SSS, clearly identifies the compact object in this system as a WD. The proposed optical counterpart, 2MASS J01151085-7256102, has a spectral type of O9IIIe, suggesting that Swift J011511.0-725611 is in fact a newly discovered BeWD binary system. Swift J011511.0-725611 would only be the sixth candidate BeWD identified, fourth in the SMC, following on from Swift J004427.3-734801 which was also discovered by S-CUBED, XMMU J010147.5-715550 (Sturm et al. 2012), Suzaku J0105-72 (Cracco et al. 2018), and the two Large Magellanic Cloud (LMC) BeWD candidates, XMMUJ 052016.0-692505 (Kahabka et al. 2006) and RX J0527.8-6954 (Oliveira et al. 2010).

Fig. 9 shows the strong correlation between the brightness of 2MASS J01151085-7256102 and the redness of the whole system. The observed light is arising as a result of a fixed contribution from the $\mathrm{OB}$ star plus a variable contribution from the, generally cooler, circumstellar disc. Taking the spectral class of the star to be O9IIIe (Golden-Marx et al. 2016) with a colour of (V-I) $=-0.37$ (Pecaut \& Mamajek 2013), and assuming the reddening to the SMC to be $\mathrm{E}(\mathrm{V}-\mathrm{I})=0.067$ (Skowron et al. 2021), this implies that the underlying observed colour of the star, without any circumstellar disc, would be $(\mathrm{V}-\mathrm{I}) \sim-0.30$. It is clear from Fig. 4 that the overall colour never reaches that blue extreme during the time of these OGLE observations, indicating the continual presence of a significant, but variable circumstellar disc in this system.

Kennea et al. (2020) reported that in the case of the S-CUBED discovered BeXRB Swift J004516.6-734703, the UVOT $u v w 1$ brightness tracked the OGLE- $I$ brightness. The OGLE- $I$ band light-curve in Fig. 6 taken between the June 2016 and March 2020 show that the source not showing any large scale variations during this period (excluding orbital variations). Similarly the UVOT $u v w 1$ light-curve (Fig. 3) is steady during this period and after, including the period of the X-ray outburst. If $u v w l$ brightness is a proxy for $I$-band, then this is suggestive that the optical state remained stable after March 2020. We note that no UV enhancement is expected associate with the super-soft X-ray emission, as the extrapolated UV brightness is too faint to be detected. 


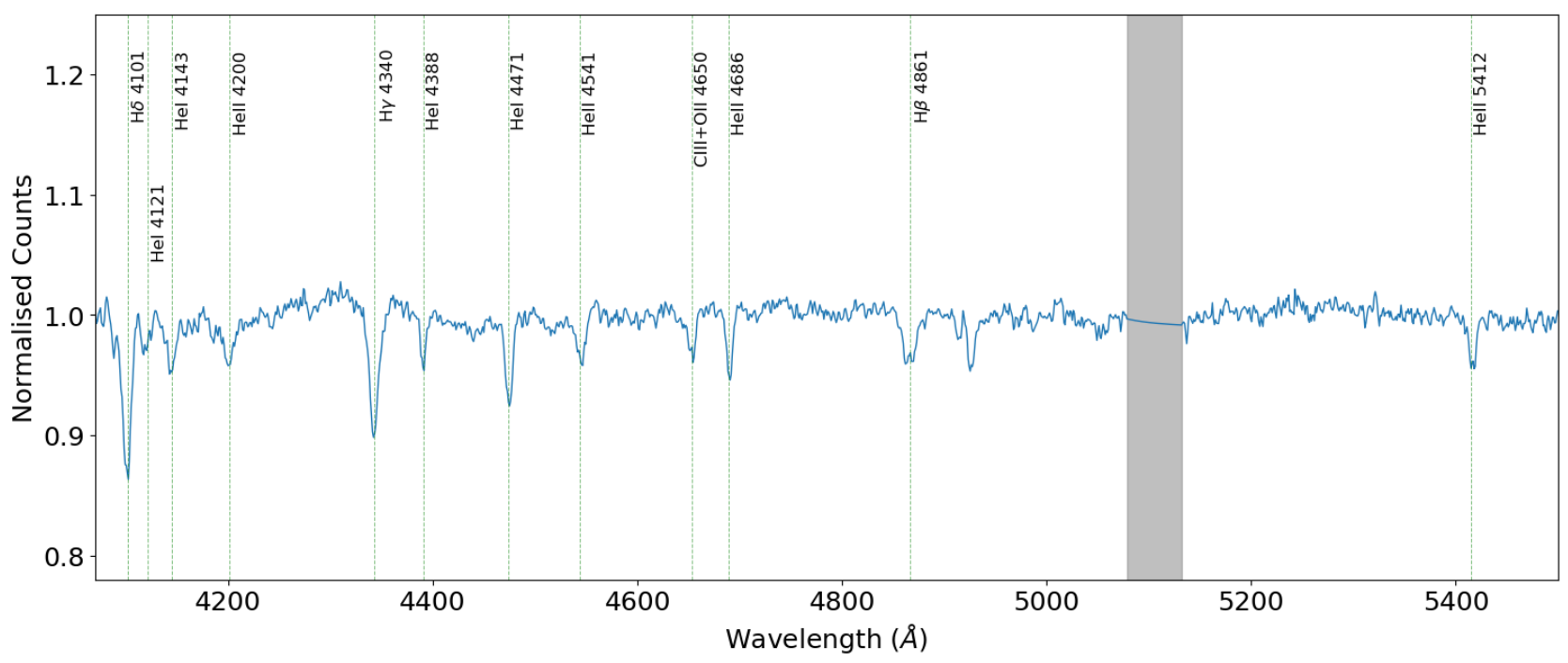

Figure 8. SALT blue end spectrum of 2MASS J01151085-7256102. The grey vertical column indicates a chip gap, which has been interpolated across. Prominent hydrogen, helium and ionized helium lines are visible, and are discussed further in the text.

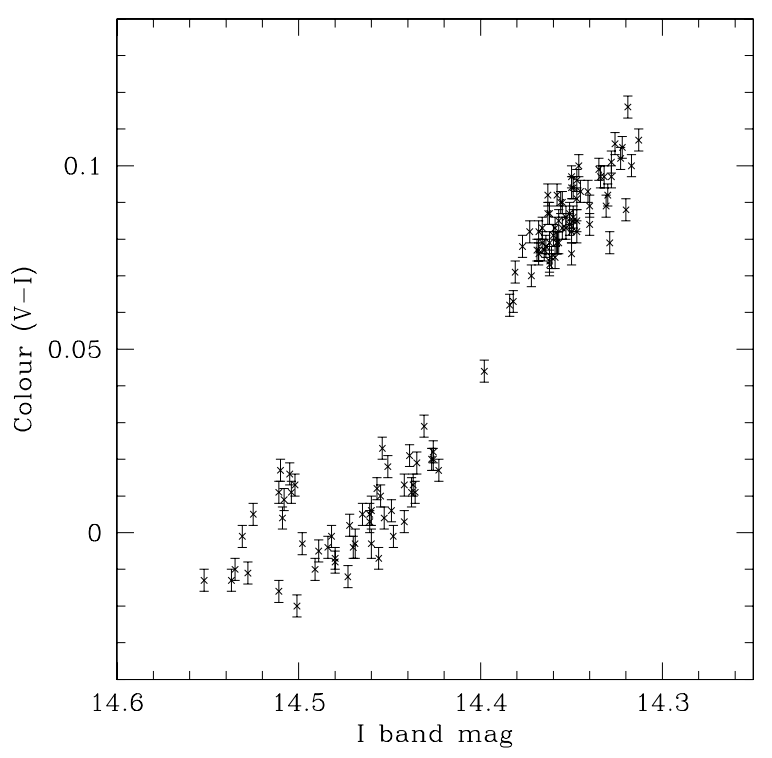

4

Figure 9. Colour-magnitude trend diagram from the OGLE data of 2MASS J01151085-7256102.

The timings of all the XRT detections in Swift J011511.0-725611 were investigated, as was the strength of each detection. Folding both against the phase given in Eqn. 1 does not reveal any preferences for $\mathrm{X}$-ray emission at any specific binary phase. This is, perhaps, not surprising if we are seeing a classic Type II outburst. Such events are believed to occur when the circumstellar disc expands sufficiently far to encompass the whole of the neutron star orbit. Type-I outbursts, if they exist in these BeWD binary systems, are likely below the level of detection of the shallow S-CUBED exposures.

The small size of the $\mathrm{H} \alpha$ emission seen in this system puts it amongst a group of other High Mass X-ray Binary (HMXB) systems which also show a small Equivalent Width $(\geq-10 \AA)$, but still exhibit significant X-ray activity. There are five such systems known in the SMC (SXP 8.65, SXP 8.80, SMC X-1, SXP 101, SXP 2.37) and one in the Milky Way (SAX J2103.5+4545) that have well documented small $\mathrm{H} \alpha \mathrm{EW}$ values and known binary periods. Following the technique described in Coe \& Kirk (2015) it is possible to estimate the size of the circumstellar disks \& the size of the orbital radii, and then compare these values with that found here for Swift J011511.0725611. The technique relies upon estimating the circumstellar disk size from the strength of the $\mathrm{H} \alpha$ emission, and the size of the orbit from the orbital period and the mass of the OB star. Using that approach for Swift J011511.0-725611 the circumstellar disk size is estimated to be $(3.35 \pm 0.14) \times 10^{10} \mathrm{~m}$ and the orbital size to be $(5.47 \pm 0.17) \times 10^{10} \mathrm{~m}$. This result is shown in Fig. 10 .

It is immediately apparent that a strong correlation exists between these two parameters, confirming that in these tight orbital situations the orbiting compact object plays a major role in constraining the extent of the circumstellar disc. Such restrictions have been proposed by Okazaki \& Negueruela (2001) based upon their Smooth Particle Hydrodynamic simulations. Though the compact object in this system is proposed here to be a WD rather than a NS for the other systems, the location of Swift J011511.0-725611 on this diagram strongly suggests that such an object can also play a very similar role to that of the neutron star in the other systems. However, studies of further Be white dwarf systems are needed to confirm that no significant differences actually exists.

A large population of BeWDs has been predicted by binary evolution modeling (Raguzova 2001), however despite this relatively few such systems are known. Swift J004427.3-734801 became the only third such system to be discovered in the SMC (Coe et al. 2020), despite the apparent overabundance of BeXRBs compared to the Milky Way. The fourth discovered SMC BeWD, Swift J011511.0-725611 shares similar properties to Swift J004427.3-734801, with orbital periods of $\sim 20$ days, and both being discovered through apparent Type-II X-ray outbursts.

It is clear that without a sensitive, soft-band regular X-ray survey of the SMC such as S-CUBED, the likelihood of discovery of sources like Swift J011511.0-725611 and Swift J004427.3-734801, is much smaller. The peak fluxes of these sources, combined with their soft spectra put them below the level of detection of current all-sky survey instruments such as MAXI (Matsuoka et al. 2009), Fermi/GBM (Meegan et al. 2009) and Swift/BAT (Barthelmy et al. 2005). 


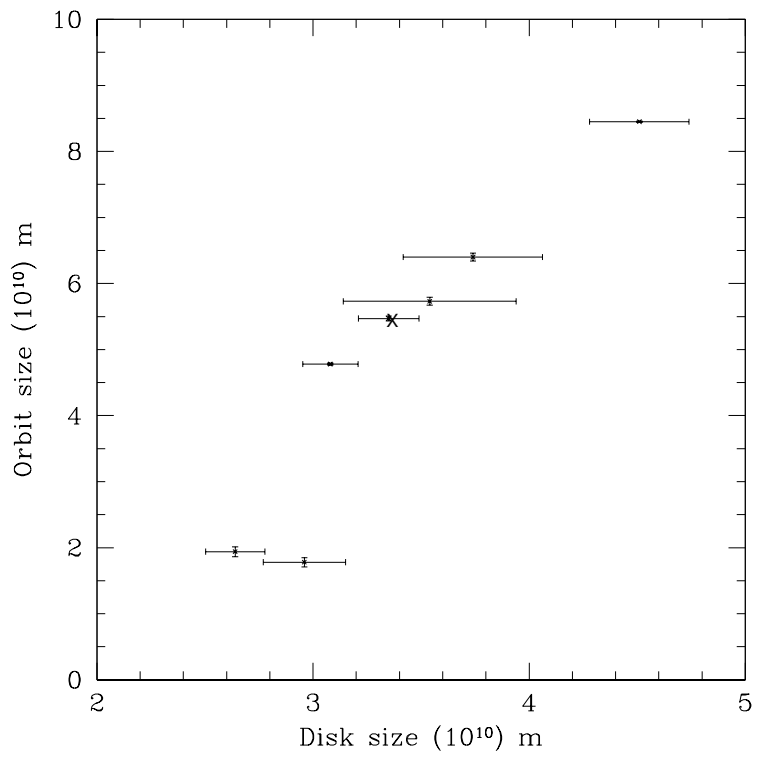

Figure 10. The estimated circumstellar disc size versus orbital size for a sample of HMXB systems, plus that of Swift J011511.0-725611 (shown as a $\mathrm{X}$ symbol). See text for details.

If Type-I outbursts are absent or too faint to be detected by SCUBED in SMC BeWDs, to detect X-ray emission from a BeWD it needs to be undergoing a Type-II outburst, which are brighter and longer lasting than Type-I. Unfortunately Type-II outbursts are also much less frequent, with often years between outbursts. So in order to discover new BeWDs, observations need to occur in the right place, i.e. in a location where BeWDs are likely to exist and are detectable (i.e. low absorption in the line of sight), and at the right time (when they are undergoing a Type-II outburst). Given this, the relative paucity of known BeWDs can at least partially be explained due to observational bias.

This highlights the importance of sensitive soft X-ray surveys as a discovery engine of BeWD binaries. It is therefore not surprising that Swift J004427.3-734801 was also detected (Haberl et al. 2020) by the all-sky soft X-ray survey telescope eROSITA (Predehl et al. 2021). However, the focused SMC only approach of S-CUBED has proven a powerful tool for discovery of BeWD, by in 5 years doubling the known number of SMC BeWDs.

It is notable that as yet, a BeWD has not been discovered in the Milky Way, only in the SMC and LMC. This may be a selection effect due to much higher extinction rates in the plane of the Milky Way, combined with the observational biases discussed above. On the other hand the different metallicity of the Magellanic Clouds may play a crucial role in the evolution of such systems.

We note that BeWD are important systems, as they have been suggested as progenitors of peculiar overluminous Type Ia, and possibly also super luminous Type II supernovae such as SN 2006gy Ablimit (2021). In addition they may also play a role as progenitors in the evolution of the proposed BH - WD systems (Shao \& Li 2021). In the example presented in this paper, and in the similar BeWD source studied in our previous paper (Coe et al. 2020), the mass of the OB star presents a real challenge in understanding how so much mass transfer would have taken place to evolve these BeWD systems. It is clearly crucial that future observations establish an accurate estimate of their numbers in the family of evolved binary populations.

\section{CONCLUSIONS}

We report in this work the discovery, by S-CUBED of a new BeXRB containing a WD compact object, Swift J011511.0-725611, which underwent a Type-II X-ray outburst over a period of 120 days. Analysis of optical and IR data from Swift, OGLE and SALT confirm the presence of a O9IIIe companion star hosting a CSD, and X-ray data are consistent with a WD at SMC distance. BeWD binary systems are a rare class of object, of which Swift J011511.0-725611 is only the fourth such system detected in the SMC, despite the predicted large population. We suggest that this may be due in part to the super-soft $\mathrm{X}$-ray emission being only detectable using sensitive focused X-ray imaging telescopes, and only during relatively infrequent Type-II outbursts, that typically last of the order of months. This means that the likelihood of discovery of BeWD sources is greatly increased with regular sensitive soft X-ray monitoring, such as that performed by S-CUBED. It is likely that all-sky sensitive soft X-ray monitoring performed by eROSITA will be a significant discovery engine for BeWD binaries, perhaps discovering the first such system in the Milky Way. However, in order to increase the number of confirmed BeWD binaries, continued monitoring of the SMC by surveys like S-CUBED will prove invaluable.

\section{ACKNOWLEDGEMENTS}

The OGLE project has received funding from the National Science Centre, Poland, grant MAESTRO 2014/14/A/ST9/00121 to AU. JAK and ZAC acknowledge support from NASA Grant NAS5-00136. PAE acknowledges UKSA support. LJT is supported by the South African National Research Foundation. Some of the observations reported in this paper were obtained with the Southern African Large Telescope (SALT) under programme 2019-2-MLT-004. The Polish participation in SALT is funded by grant no. MNiSW DIR/WK/2016/07. This work made use of data supplied by the UK Swift Science Data Centre at the University of Leicester.

\section{DATA AVAILABILITY}

The data underlying this article will be shared on reasonable request to the corresponding author.

\section{REFERENCES}

Ablimit I., 2021, PASP, 133, 074201

Arnaud K. A., 1996, in Jacoby G. H., Barnes J., eds, Astronomical Society of the Pacific Conference Series Vol. 101, Astronomical Data Analysis Software and Systems V. p. 17

Barthelmy S. D., et al., 2005, Space Sci. Rev., 120, 143

Burgh E. B., Nordsieck K. H., Kobulnicky H. A., Williams T. B., O'Donoghue D., Smith M. P., Percival J. W., 2003, in Iye M., Moorwood A. F. M., eds, Proc. SPIEVol. 4841, Instrument Design and Performance for Optical/Infrared Ground-based Telescopes. pp 1463-1471, doi: $10.1117 / 12.460312$

Burrows D. N., et al., 2005, Space Sci. Rev., 120, 165

Coe M. J., Kirk J., 2015, MNRAS, 452, 969

Coe M. J., Kennea J. A., Evans P. A., Udalski A., 2020, MNRAS, 497, L50

Cracco V., Orio M., Ciroi S., Gallagher J., Kotulla R., Romero-Colmenero E., 2018, ApJ, 862, 167

Dickey J. M., Lockman F. J., 1990, ARA\&A, 28, 215

Evans P. A., et al., 2009, MNRAS, 397, 1177

Gehrels N., et al., 2004, ApJ, 611, 1005

Goad M. R., et al., 2007, A\&A, 476, 1401 
Golden-Marx J. B., Oey M. S., Lamb J. B., Graus A. S., White A. S., 2016, ApJ, 819, 55

Graczyk D., et al., 2014, ApJ, 780, 59

Haberl F., Pietsch W., 2004, A\&A, 414, 667

Haberl F., Sturm R., 2016, A\&A, 586, A81

Haberl F., Filipović M. D., Pietsch W., Kahabka P., 2000, A\&AS, 142, 41

Haberl F., et al., 2020, The Astronomer's Telegram, 13709

Hanuschik R. W., Hummel W., Sutorius E., Dietle O., Thimm G., 1996, A\&AS, 116, 309

Kahabka P., 2006, Advances in Space Research, 38, 2836

Kahabka P., Haberl F., Payne J. L., Filipović M. D., 2006, A\&A, 458, 285

Kennea J. A., Coe M. J., Evans P. A., Waters J., Jasko R. E., 2018, ApJ, 868, 47

Kennea J. A., Coe M. J., Evans P. A., Monageng I. M., Townsend L. J., Siegel M. H., Udalski A., Buckley D. A. H., 2020, MNRAS, 499, L41

Kennea J. A., Coe M. J., Evans P. A., Townsend L. J., Van Wyk F., Udalski A., 2021, The Astronomer's Telegram, 14341

Lamb J. B., Oey M. S., Graus A. S., Adams F. C., Segura-Cox D. M., 2013, ApJ, 763, 101

Lomb N. R., 1976, Ap\&SS, 39, 447

Matsuoka M., et al., 2009, PASJ, 61, 999

Meegan C., et al., 2009, ApJ, 702, 791

Mukai K., 2017, PASP, 129, 062001

Munar-Adrover P., Paredes J. M., Ribó M., Iwasawa K., Zabalza V., Casares J., 2014, ApJ, 786, L11

Negueruela I., Okazaki A. T., Fabregat J., Coe M. J., Munari U., Tomov T., 2001, A\&A, 369, 117

Okazaki A. T., Negueruela I., 2001, in White N. E., Malaguti G., Palumbo G. G. C., eds, American Institute of Physics Conference Series Vol. 599, X-ray Astronomy: Stellar Endpoints, AGN, and the Diffuse X-ray Background. pp 810-813, doi:10.1063/1.1434748

Oliveira A. S., Steiner J. E., Ricci T. V., Menezes R. B., Borges B. W., 2010, A\&A, 517, L5

Pecaut M. J., Mamajek E. E., 2013, ApJS, 208, 9

Porter J. M., Rivinius T., 2003, PASP, 115, 1153

Predehl P., et al., 2021, A\&A, 647, A1

Pringle J. E., Webbink R. F., 1975, MNRAS, 172, 493

Raguzova N. V., 2001, A\&A, 367, 848

Reig P., 2011, Ap\&SS, 332, 1

Roming P. W. A., et al., 2005, Space Sci. Rev., 120, 95

Scargle J. D., 1982, ApJ, 263, 835

Shao Y., Li X.-D., 2021, arXiv e-prints, p. arXiv:2107.03565

Shimura T., 2000, MNRAS, 315, 345

Skowron D. M., et al., 2021, ApJS, 252, 23

Sturm R., Haberl F., Pietsch W., Coe M. J., Mereghetti S., La Palombara N., Owen R. A., Udalski A., 2012, A\&A, 537, A76

Townsend L. J., Kennea J. A., Coe M. J., McBride V. A., Buckley D. A. H., Evans P. A., Udalski A., 2017, MNRAS, 471, 3878

Udalski A., 2003, Acta Astron., 53, 291

Udalski A., Szymański M. K., Szymański G., 2015, Acta Astron., 65, 1

Walborn N. R., Fitzpatrick E. L., 1990, PASP, 102, 379

Wilms J., Allen A., McCray R., 2000, ApJ, 542, 914

This paper has been typeset from a $\mathrm{T}_{\mathrm{E}} \mathrm{X} / \mathrm{L} \mathrm{T} \mathrm{E} \mathrm{X}$ file prepared by the author. 